\title{
Aortic aneurysm with complete atrioventricular block and acute coronary syndrome
}

\author{
Moacyr Magno Palmeira1,2,3*, Hellen Yuki Umemura Ribeiro², Yan Garcia Lira², \\ Fernando Octávio Machado Jucá Neto², Ivone Aline da Silva Rodrigues², Maitê Silva Martins Gadelha² \\ and Yuri Santana do Carmo ${ }^{2}$
}

\begin{abstract}
Background: Acute aortic dissection (AAD) is a highly lethal and prevalent cardiovascular emergency. AAD can develop into atrioventricular conductivity disorders caused by coronary artery dissection, with acute myocardial infarction (AMI) being the most frequent clinical sign. In many deceased patients, the diagnosis is not confirmed until autopsy, and $85 \%$ receive the wrong therapy as a result of misdiagnosis.

Case presentation: A 49-year-old male patient presenting with prolonged, intense and sharp precordial pain radiating to his back, as well as cold sweats, nausea and vomiting, was admitted to the cardiac emergency service. Thorax examination revealed normal bilateral breath sounds and a respiratory frequency of 24 incursions/min ( $\mathrm{SpO}_{2} 97 \%$ ). Cardiac auscultation revealed a heartbeat that was rhythmic, regular, and bradycardic. There was a visible highintensity pulsation in the suprasternal notch, a diastolic murmur audible at the aortic focus, and a fourth heart sound on auscultation. The patient was diagnosed with Stanford type A AAD, concomitant complete atrioventricular block, and impairment of the right coronary artery, progressing to acute coronary syndrome (ACS) and spontaneous rupture of the aortic aneurysm. After a hemodynamic study, the patient was transferred for urgent surgical treatment and passed away during the procedure.
\end{abstract}

Conclusion: Physical examination is essential to be able to disregard AAD as the main cause of AMI. The consequences of a misdiagnosis can be fatal if thrombolytic or anticoagulant therapy is chosen as the initial treatment; therefore, surgery is the best treatment for aortic dissection.

Keywords: Cardiovascular diseases, Aortic aneurysm, Acute coronary syndrome, Atrioventricular block

\section{Background}

Acute aortic dissection (AAD) is a highly lethal cardiovascular emergency with an incidence of 2000 new cases per year in the United States and 3000 in Europe. The rate of incidence is 5-30 per million people per year and the mortality rate is $3.2 / 100.000$ per year [1].

Atrioventricular conductivity disorders caused by coronary artery dissection involving $\mathrm{AAD}$ are rare complications $[2,3]$. Both coronary arteries can be affected, and

\footnotetext{
*Correspondence: palmeiramm@ig.com.br

${ }^{3} 60$ Amazonas square, Jurunas, Belém, Pará 66025-070, Brazil

Full list of author information is available at the end of the article
}

acute myocardial infarction (AMI) is the most frequent clinical symptom [2].

We report a case of Stanford type A AAD with right coronary artery dissection, which developed into complete atrioventricular block and acute coronary syndrome (ACS).

\section{Case presentation}

A 49-year-old male patient, a farmer, married, and living in Irituia City, presented at the cardiac emergency service with prolonged, intense, and sharp precordial pain radiating to his back. He also had intense cold sweats, nausea, and vomiting. The symptoms started $6 \mathrm{~h}$ prior to hospital 
admission. The patient was obese, hypertensive, and a long-time smoker. He was taking $25 \mathrm{mg}$ of captopril daily. Diabetes, alcoholism, previous AMI, cerebrovascular accident, or any other comorbidity were denied. He was admitted in good general condition, hydrated, pale $(++/ 4+)$, acyanotic, afebrile, mildly dyspneic, bradycardic, conscious, oriented in space and time, verbalizing well, and walking with assistance.

A thorax examination revealed normal bilateral breath sounds without adventitious sounds and a respiratory frequency of 24 incursions/min $\left(\mathrm{SpO}_{2} 97 \%\right)$. Cardiac auscultation yielded a rhythmic, regular, and bradycardic heart rate ( 44 beats/min). There was a visible high-intensity pulsation in the suprasternal notch, a diastolic murmur audible at the aortic focus, and a fourth heart sound on auscultation. He had asymmetry of pulses in the upper limbs, with the right radial artery pulse stronger than the left. Blood pressure was 120/80 mmHg. His abdomen was distended and painful during examination. Bowel sounds were present on auscultation.

An electrocardiogram (ECG) revealed complete atrioventricular block and ST-segment elevation in the inferior wall. Therefore, the patient was treated for AMI using supportive measures, such as oxygen and initial thrombolytic therapy with tenecteplase $45 \mathrm{mg}$ in a single bolus, to achieve reperfusion. After stabilization, the patient was sent for hemodynamic evaluation for urgent catheterization.

Coronary angiography showed an aortic dissecting aneurysm (Fig. 1) and a right coronary false lumen area (Fig. 2). The patient was diagnosed with Stanford type A AAD, concomitant complete atrioventricular block, and impairment of the right coronary artery, progressing to ACS and spontaneous rupture of the aortic aneurysm.

After a hemodynamic study, the patient was transferred for emergency surgical treatment. The surgery was performed through a median sternotomy. The initial inspection revealed aortic dilatation and visible aortic dissection in the aortic crest as well as in the proximal portion. There was an aneurysm rupture in the aortic right proximal portion with remnants clots. During these initial steps, the patient's blood pressure decreased and failed to stabilize. Further, several bleeding foci presented and aortic cross-clamping was difficult due to dissection damage. The patient died during the procedure.

\section{Discussion}

AAD is an acute lesion of the aortic wall, accompanied by separation of the intimal and medial layers of this artery, due to rupture or intramural hematoma [1]. It is a very serious vascular event with a high mortality rate. Further, many deceased patients remain misdiagnosed until

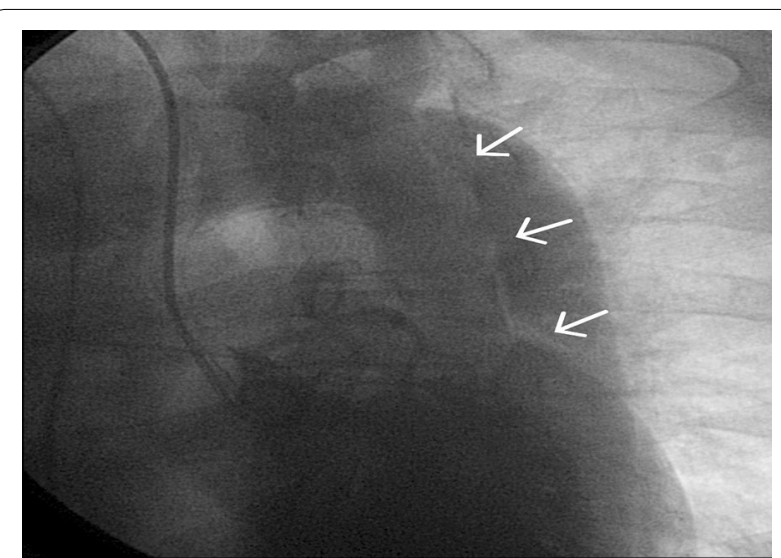

Fig. 1 Aortic dissecting aneurysm. Coronary angiography revealed an aortic dissecting aneurysm during hemodynamic evaluation for urgent catheterization

autopsy. Therefore, a rapid diagnosis is vital to providing the appropriate treatment in a timely manner [2]. The dissection begins transversely, as it is located in the ascending aorta in $70 \%$ of cases, with $20 \%$ in the descending aorta after the aortic arch and $10 \%$ in the transverse aortic arch. According to the Stanford classification, aneurysms in the ascending aorta are type A and aneurysms not involving the ascending aorta are type B [3]. Once started, further dissection can be distal, retrograde, or can occur in both directions, advancing in variable lengths [4].

The main symptom of AAD is severe chest pain, usually in the anterior region. The pain commonly has an abrupt onset and is of high intensity, usually maximum from the beginning of the dissection [5]. Similarly to AMI, acute dissection is usually associated with symptoms of profuse sweating, pallor, tachycardia, tremors, nausea, and vomiting [5].

ECG in AAD is often nonspecific, with approximately a third of them revealing changes that are consistent with

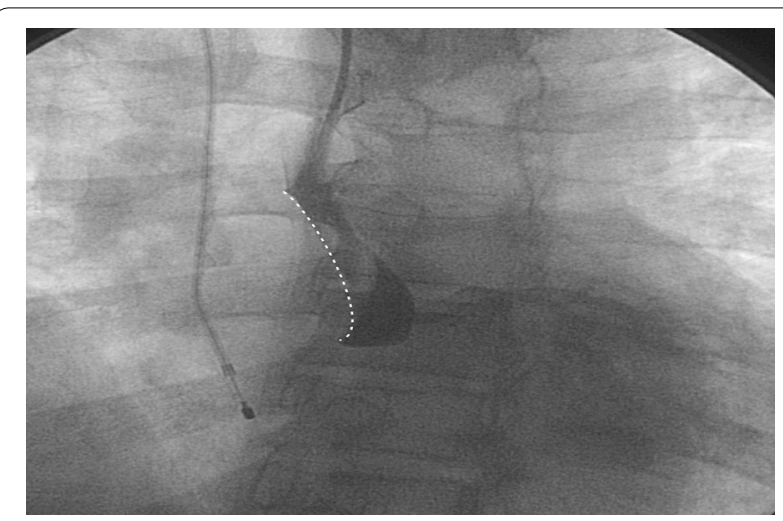

Fig. 2 Right false coronary lumen area. Coronary angiography revealed a right coronary false lumen area caused by aortic dissecting aneurysm extending to the right coronary ostium 
left ventricular hypertrophy and another third appearing normal. Nevertheless, obtaining an ECG is important for three reasons. First, in patients with AAD presenting with unspecified chest pain, the absence of $T$ waves and ST-segment ischemia may suggest another main diagnosis, aside from myocardial ischemia and including AAD, in patients with proximal dissection. Second, the ECG may reveal AMI when the dissection flap involves a coronary artery [6]. Third, it can reveal the coexistence of a cardiac rhythm disorder [7].

In AAD, rare and irregular rhythms may be present, such as bundle branch block and atrioventricular block. In Stanford type A dissections, it is suggested that the pathophysiological mechanism, which generates the rhythm disorder, is the presence of hematoma in the interatrial septum and/or in the atrioventricular junction, which results in cardiac block [7].

In our case, the ECG revealed complete atrioventricular block and changes suggestive of AMI with inferior wall involvement. Coronary artery dissection in the course of AAD is usually diagnosed by the presence of AMI or by identifying the displacement of the intimal layer through coronary angiography [5]. In a small minority, approximately $1-2 \%$ of the cases [8], a proximal dissection flap may involve the ostium of a coronary artery and cause myocardial infarction, which can hinder the diagnosis. The dissection more often affects the right coronary artery than the left, explaining why these myocardial infarctions tend to have a lower location [6]. Unfortunately, when there is a secondary AMI, the symptoms may lead to a misunderstanding of the primary symptoms of $\mathrm{AAD}$ and complicate the clinical picture. The possibility that within the electrocardiographic evidence of myocardial infarction, the existing AAD may remain unrecognized is even more worrisome. Therefore, physical examination is essential to be able to disregard AAD as the main cause of AMI. Asymmetry of the peripheral pulses is found in $50 \%$ of type A dissections. A diastolic murmur audible at the aortic focus with normal valves contributes to an AAD diagnosis, and the presence of abdominal sounds, despite the abdomen being distended and painful, could suggest that there is no involvement of the descending aorta or global damage[3-5].

The diagnosis of AMI and AAD can occur concomitantly in $1.5-7.5 \%$ of cases. As surgery is the best treatment for $\mathrm{AAD}$, the consequences of a misdiagnosis within this syndrome can be fatal if thrombolytic therapy is chosen as the primary treatment. Moreover, $85 \%$ of the patients receive the wrong therapy as a result of misdiagnosis $[2,4-6]$. In a literature review, among 21 cases of $\mathrm{AAD}$ that were treated with thrombolytic therapy, early mortality was reported in $71 \%$ cases, of which many deaths were caused by cardiac tamponade [9].

The very high risk of cardiac tamponade and other complications has led to a consensus that surgery is superior to clinical management for acute type A dissections. While surgical repair of the ascending aorta greatly reduces such risk, some of the patients referred to surgery are already in a very serious condition, which reduces their chances of survival, not because of surgical complications but as a result of their serious medical condition [2].

\section{Conclusion}

As the symptoms of a secondary AMI can lead to a misunderstanding of the primary symptoms of AAD, physical examination is essential in being able to disregard AAD as the main cause of AMI. The consequences of a misdiagnosis can be fatal if thrombolytic or anticoagulant therapy is chosen as the initial treatment. Therefore, surgery is the best treatment for aortic dissection.

\section{Abbreviations}

AAD: acute aortic dissection; ACS: acute coronary syndrome; AMI: acute myocardial infarction; ECG: electrocardiogram.

\section{Authors' contributions}

MMP conceived and designed the experiment. HYUR, YGL, FOMJN, IASR MSMG, YSC participated in sample collection and performed the experiments. MMP was involved in patient care. MMP, HYUR, YGL, FOMJN, IASR, MSMG, YSC contributed drafting the manuscript and in the transcription. MMP, HYUR, FOMJN contributed significantly in the critical revision of the manuscript. MMP, HYUR, YGL, FOMJN, IASR, MSMG, YSC designed and participated in the review and final approval of the manuscript. All authors read and approved the final manuscript.

\section{Authors' information}

MMP is Ph.D. in Biological Sciences from Universidade Federal do Estado do Pará, Master's at Cardiology from Universidade Federal de São Paulo, Specialist at Cardiology from Sociedade Brasileira de Cardiologia, Professor and

Coordinator of the Cardiology Care at Universidade do Estado do Pará, Doctor at the department of Urgency and Emergency at FHCGU/PA. HYUR is medical student at Universidade do Estado do Pará. YGL is medical student at Universidade do Estado do Pará. FOMJN is medical student at Universidade do Estado do Pará. IASR is medical student at Universidade do Estado do Pará. MSMG is medical student at Universidade do Estado do Pará. YSC is medical student at Universidade do Estado do Pará.

\section{Author details}

${ }^{1}$ Division of Cardiology, Section of Urgency and Emergency, Department of Intensive Care, Fundação Pública Estadual Hospital de Clínicas Gaspar Vianna, 2000 Alferes Costa street, Pedreira, Belém, Pará 66087-660, Brazil. ${ }^{2}$ Division of Cardiology, Section of Urgency and Emergency, Department of Clinical Care, Universidade do Estado do Pará, 2623 Perebebuí street, Marco, Belém, Pará 66087-670, Brazil. $^{3} 60$ Amazonas square, Jurunas, Belém, Pará 66025-070, Brazil.

\section{Acknowledgements}

This study did not receive any funding support. The authors would like to thank the medical staff of the hospital Fundação Pública Estadual Hospital de Clínicas Gaspar Vianna for their support in patient care. 


\section{Availability of data and materials}

All relevant data are within the paper and its supporting information files.

\section{Competing interests}

The authors declare that they have no competing interests and have received no specific funding for this work.

\section{Ethics and consent}

This work was approved by the ethics committee "Fundação Pública Estadual Hospital de Clínicas Gaspar Vianna" and a written informed consent to participate was obtained from the patient and a written informed consent to publish this case report and any accompanying images was obtained from the wife of the patient.

Received: 11 September 2015 Accepted: 19 April 2016

Published online: 04 May 2016

\section{References}

1. Buivydaite K, Semenaite V, Brazdzionyte J, Macas A. Aortic dissection. Medicina (Kaunas). 2008:44:247-55.

2. Upadhye S, Schiff K. Acute aortic dissection in the emergency department: diagnostic challenges and evidence-based management. Emerg Med Clin North Am. 2012. doi:10.1016/j.emc.2011.12.001.
3. Lionakis N, Moyssakis I, Gialafos E, Dalianis N, Votteas V. Aortic dissection and third-degree atrioventricular block in a patient with a hypertensive crisis. J Clin Hypertens (Greenwich). 2008;10:69-72.

4. Martin JFV, Andrade LG, Loureiro AAC, Godoy MF, Braile DM. Infarto agudo do miocárdio e dissecção aguda de aorta: um importante diagnóstico diferencial. Revista Brasileira de cirurgia cardiovascular. 2004;19:386-90.

5. Güvenç TS, Erer HB, Cetin R, Hasdemir H, Ilhan E, Türkkan C, et al. Acute aortic regurgitation with myocardial infarction: an important clue for aortic dissection. J Emerg Med. 2013;44:e5-8.

6. Isselbacher EM, Eagle KA, Desanctis RW. Doenças da aorta. In: Braunwald, editor. Tratado de medicina cardiovascular. São Paulo: Roca; 1999. p 1655-89.

7. Thiene $G$, Rossi L, Becker A. The atrioventricular conduction system in dissecting aneurysm of the aorta. Am Heart J. 1979;98:447-52.

8. Glower DD, Speier RH, White WD, Smith LR, Rankin JS, Wolfe WG. Management and long-term outcome of aortic dissection. Ann Surg. 1991:214:31-41.

9. Kamp TJ, Goldschmidt-Clermont PJ, Brinker JA, Resar JR. Myocardial infarction, aortic dissection, and thrombolytic therapy. Am Heart J. 1994;128:1234-7.

\section{Submit your next manuscript to BioMed Central and we will help you at every step:}

- We accept pre-submission inquiries

- Our selector tool helps you to find the most relevant journal

- We provide round the clock customer support

- Convenient online submission

- Thorough peer review

- Inclusion in PubMed and all major indexing services

- Maximum visibility for your research

Submit your manuscript at www.biomedcentral com/submit 